\title{
Relação entre Desempenho Neurocognitivo e Composição Corporal de Homens e Mulheres Expostos a Solventes Orgânicos
}

\author{
Ana Raquel Oliveira*, 1 \\ Orcid.org/0000-0002-9989-0255 \\ Thayro Andrade Carvalho ${ }^{2}$ \\ Orcid.org/0000-0001-5107-0074 \\ Natanael Antonio dos Santos ${ }^{3}$ \\ Orcid.org/0000-0001-7708-9929
}

\author{
${ }^{1}$ Universidade Federal do Piauí, Teresina, PI, Brasil \\ ${ }^{2}$ Universidade Federal do Rio Grande do Norte, Natal, RN, Brasil \\ ${ }^{3}$ Universidade Federal da Paraíba, João Pessoa, PB, Brasil
}

\section{Resumo}

Investigou-se o efeito dos solventes orgânicos sobre o desempenho neurocognitivo de homens e mulheres com e sem exposição crônica a solventes orgânicos, e verificou correlações entre o desempenho neurocognitivo e índices de composição corporal. Participaram 14 homens e 14 mulheres expostos e 14 homens e 14 mulheres não expostos. Utilizou-se o Trail Making Test (TMT) A e B, Figura Complexa de Rey (FCR), Cópia e Memória, questionário sociodemográfico e avaliação de bioimpedância. A comparação entre mulheres expostas e não expostas mostrou diferença significativa em todos os testes. A comparação entre os homens expostos e não expostos mostrou diferença significativa no TMT-A, FCRCópia, FCR-Memória. Contudo, na comparação entre homens e mulheres expostos os resultados não mostraram diferenças significantes. Já na correlação entre o desempenho neurocognitivo com índices de composição corpórea, as mulheres expostas apresentaram correlação significante entre FCR-Cópia com água corporal, peso, massa gorda, massa magra corporal. Os homens expostos apresentaram correlação significante entre o TMT-B com a Relação Cintura-Quadril e gordura visceral. No geral, os solventes orgânicos podem afetar o desempenho neurocognitivo e os efeitos da exposição crônica dependem da precisão do teste neurocognitivo e do índice de composição corpórea.

Palavras-chave:Atenção,memória, funçõesexecutivas, solventesorgânicos,composiçãocorporal,sexos.

\section{Relationship between Neurocognitive Performance and Body Composition of Men and Women Exposed to Organic Solvents}

\begin{abstract}
It was investigate the effect of organic solvents on the neurocognitive performance of men and women with and without chronic exposure to organic solvents and verify correlations between neurocognitive

* Endereço para correspondência: Universidade Federal do Piauí, Departamento de Fundamentos da Educação, Campus Ministro Petrônio Portella, s/n, Ininga, Teresina, PI, Brasil 64049-550. Fone: (86) 3215-5513. E-mail: ana-raqueloliveira@hotmail.com, thayrocarvalho@gmail.com e natanael_labv@yahoo.com.br
\end{abstract}


performance and body composition indices. Participants included 14 men and 14 exposed women and 14 men and 14 unexposed women. The Trail Making Test (TMT) A and B, the Rey Complex Figure (RCF; Copy and Memory), sociodemographic questionnaire, and bioimpedance evaluation were used. There was a significant difference between exposed and non-exposed women in all tests. Comparison between exposed and non-exposed men showed a significant difference in TMT-A, RCF-Copy, and RCF-Memory. However, there was no significant difference between sexes. Regarding the comparison between neurocognitive performance and body composition indices, for exposed women, there was a significant correlation between RCF- Copy and water, body weight, fat mass, and lean mass. Regarding the exposed men, there was a significant correlation between TMT-B and WHR (waist-hip ratio) and visceral fat. In general, organic solvents can affect neurocognitive performance and the effects of chronic exposure depend on the accuracy of the neurocognitive test and the body composition index.

Keywords: Attention, memory, executive functions, organic solvents, body composition, sexes.

\section{Relación entre Desempeño Neurocognitivo y Composición Corporal de Hombres y Mujeres Expuestos a Solventes Orgánicos}

\section{Resumen}

Se investigo el efecto de solventes orgánicos sobre el rendimiento neurocognitivo de hombres y mujeres con y sin exposición crónica a solventes orgánicos, además de verificar la correlación entre el rendimiento neurocognitivo y los índices de composición corporal. Participaron 14 hombres y 14 mujeres expuestas y 14 hombres y 14 mujeres no expuestas. Se utilizaron los siguientes tests: Trail Making Test (TMT) A y B, el test Figura Compleja de Rey (FCR; Copia y Memoria), cuestionario sociodemográfico y la evaluación de bioimpedancia. Hubo diferencia significativa entre las mujeres expuestas y las no expuestas, en todos los tests. La comparación de los hombres expuestos con los que no fueron expuestos mostró diferencia significativa en el TMT-A, FCR-Copia y FCR-Memoria. Sin embargo, no hubo diferencias significativas entre los sexos. Con relación a la comparación del rendimiento neurocognitivo con los índices de composición corporal, para las mujeres expuestas hubo correlación significativa entre la FCR-Copia y el agua, peso, masa corporal grasa y masa corporal magra. Para los hombres expuestos hubo correlación significativa entre la TMT-B y la relación cintura/cadera (RCC) y grasa visceral. En general, los solventes orgánicos pueden afectar el desempeño neurocognitivo y los efectos de la exposición crónica dependen de la precisión del test neurocognitivo y del índice de composición corporal.

Palabras clave: Atención, memoria, funciones ejecutivas, solventes orgánicos, composición corporal, sexos.

Os solventes orgânicos são formados por hidrocarbonetos derivados da destilação do petróleo e utilizados na composição de tintas, cosméticos, desengordurantes, pesticidas e combustíveis (Occupational Safety and Health Service - OSHS, 1998). Estas substâncias são tóxicas para os seres humanos, pois são voláteis e lipofílicas, distribuindo-se nos órgãos ricos em tecidos gordurosos e vascularizados como o cérebro (Monat-Descamps \& Deschamps, 2012).
Pesquisas com humanos e modelos animais mostram que a exposição crônica aos solventes orgânicos pode resultar em alterações estruturais do sistema nervoso central (SNC), causando desmielinização axonal, degeneração neuronal, e perda celular (Barbosa, Boon, \& Khuu, 2015; Lotti, Bleecker, \& Aminoff, 2015), assim como alterações nas funções motoras, sensoriais e neurocognitivas (Morrow \& Scott, 2002; Sabbath et al., 2014). O declínio na capacidade 
cognitiva pode ser preditor do desenvolvimento de uma série de transtornos neurodegenerativos, como demências, por exemplo (Blazer, Yaffe, \& Liverman, 2015).

A exposição ocupacional a compostos orgânicos voláteis pode levar a 35 milhões casos de doenças anualmente (International Labour Office - ILO, 2014). De acordo com a ILO (2014) em 2011, ocorreram cerca de 888.893 mil mortes decorrentes de complicações pela exposição a substâncias químicas tóxicas, sendo 1.112 casos devido condições neuropsiquiátricas (637 mulheres e 475 homens).

Nos últimos anos, o número de mulheres contratadas como atendentes de postos de combustíveis aumentou na maioria dos países em desenvolvimento e em outras atividades industriais em países da Europa Ocidental (Ernstgård, Gullstrand, Löf, \& Johanson, 2002). Contudo, a maioria das pesquisas tem sido realizada com homens (Saygun et al., 2012; Song, Yu, \& Lao, 2015; Thetkathuek, Jaidee, Saowakhontha, \& Ekburanawat, 2015) ou animais machos (Beasley, Evansky, \& Bushnell, 2012; Zhong et al., 2013), provavelmente por que o sexo masculino ainda é maioria neste tipo de atividade (Vahter et al., 2007), ou devido a crença histórica de que o trabalho em ambientes de risco, incluindo os que lidam com solventes, é restrito aos homens (Mergler, 2012). Esses resultados acabam sendo generalizados as mulheres (Ernstgård et al., 2002) que são expostas aos mesmos limites de tolerância baseados em estudos com o sexo masculino (Kawai, Takeuchi, \& Ikeda, 2015).

Diferenças fisiológicas e bioquímicas entre os sexos têm potencial para alterar a quantidade nos tecidos e os efeitos de alguns solventes sobre a saúde (Klaassen \& Watkins, 2012). Em geral, as mulheres têm percentual de tecido adiposo maior e os homens têm mais massa muscular e peso corporal (Tseng et al., 2014).

As diferenças na composição corporal têm levantado diferentes pontos de vista sobre os efeitos dos solventes orgânicos em homens e mulheres. Do ponto de vista toxicológico, os solventes lipofílicos acumulam-se no tecido adiposo e quanto mais gordura corporal maior a quantidade e menor depuração dos agentes químicos (Klaassen \& Watkins, 2012). Assim, mulheres poderiam ser mais sensíveis do que os homens aos efeitos dos solventes, pois têm uma percentagem de gordura corporal mais elevada (Gleiter \& Gundert-Remy, 1996).

Do ponto de vista da farmacocinética e farmacodinâmica, variações entre os sexos, como peso corporal, volume plasmático, e tempo de esvaziamento gástrico podem interferir na eficácia e toxicidade das drogas (Gandhi, Aweeka, Greenblatt, \& Blaschke, 2004). Dessa forma, alguns fármacos podem ter um volume relativamente maior de distribuição e serem metabolizados mais lentamente nas mulheres em comparação aos homens (Soldin \& Mattison, 2009). Como os solventes lipofílicos com alto coeficiente de partição óleo/água são distribuídos e se concentraram na gordura corporal, esse armazenamento reduziria a concentração do toxicante no tecido-alvo; consequentemente, sua toxicidade pode ser menos severa em um indivíduo obeso do que em um indivíduo magro (Klaassen \& Watkins, 2012). Nesta perspectiva, a gordura corporal mais elevada nas mulheres poderia ser um fator de proteção (Lof \& Johanson, 1998).

Apesar disto, as diferenças fisiológicas e bioquímicas entre os sexos não têm sido consideradas no monitoramento da exposição aos solventes orgânicos (Vahter et al., 2007; Weiss, 2012). Talvez por este motivo, poucos estudos tenham comparado as diferenças entre os sexos dos efeitos neurocognitivos da exposição aos solventes orgânicos em modelos animais (Berenguer, Soulage, Perrin, Pequignot, \& Abraini, 2003; Berr et al., 2010; Malek, Möritz, \& Fanghänel, 2003) e humanos (Morrow \& Scott, 2002).

Estudo com modelos animais realizado por Malek et al. (2003) comparou o desempenho de 120 ratos (machos e fêmeas) da espécie LEW.1K expostos a diferentes concentrações de formaldeído (0.1 ppm, 0.5ppm, $5.4 \mathrm{ppm}$ ) com controles sem exposição. $\mathrm{O}$ experimento utilizava tarefa que envolvia aprendizagem e memória, além disso, analisaram a histologia de alguns 
órgãos. Durante o experimento, os animas inalavam os solventes por duas horas/dia durante dez dias consecutivos e o teste era realizado duas horas após a exposição. A tarefa do animal era encontrar a saída de um labirinto com água. $\mathrm{O}$ tempo e o número de erros foram registrados. Os pesquisadores observaram que o grupo controle necessitou de períodos de natação mais curtos e tiveram menos erros para completar o teste do labirinto de água em comparação com o grupo experimental. A comparação entre os ratos machos e fêmeas expostos revelou que as fêmeas, em geral, concluíram o teste em menos tempo que os machos. Entretanto, no grupo de maior concentração de formaldeído (5.4 ppm) não houve diferenças entre os sexos com relação ao número de erros. Os dados indicaram que o formaldeído pode afetar a aprendizagem e a memória de ratos machos e fêmeas, mesmo sem alteração clínica ou histopatológica. Segundo Spyker (1979), mudanças comportamentais podem ser consideradas sinais precoces de processos de intoxicação do organismo.

Outro estudo com modelos animais, realizado por Berenguer et al. (2003), investigou os efeitos comportamentais e neuroquímicos da inalação de $40 \mathrm{ppm}$ de tolueno em ratos Sprague-Dawley expostos (6 machos e 6 fêmeas) e controles (6 machos e 6 fêmeas). Os animais expostos eram colocados em uma câmara experimental duas vezes por semana (104 horas) durante 16 semanas. Os resultados deste estudo mostraram que a exposição subcrônica ao tolueno: (a) não alterou a atividade locomotora de ratos expostos agrupados comparados aos controles agrupados. Mas as fêmeas de ambos os grupos apresentaram atividade locomotora maior que os machos; (b) já a atividade de criação foi menor nos ratos expostos agrupados em comparação com os controles agrupados. Porém, não foi encontrada diferença significativa entre ratos machos e fêmeas tanto do grupo controle quanto do exposto; (c) a sensibilidade à narcose foi maior nos ratos expostos agrupados do que nos controles agrupados, sendo maior nos machos de ambos os grupos comparados as fêmeas de ambos os grupos; (d) aumento de 5-hydro- xytryptophan (5-HT) no hipocampo e no córtex pré-frontal de fêmeas expostas e uma diminuição de 5-HT e dihydroxyphenylalanine (DOPA) no hipocampo dos machos expostos, áreas nas quais não se esperava alteração. Em geral, a exposição subcrônica a $40 \mathrm{ppm}$ de tolueno pode afetar o funcionamento cognitivo e comportamental, mas não foi possível estabelecer relação com as alterações neuroquímicas.

Já a pesquisa com humanos desenvolvida por Berr et al. (2010) consistiu em um estudo longitudinal, avaliando se a exposição durante a vida adulta estava associada com baixo desempenho neurocognitivo no final da meia-idade. Participaram 5.242 voluntários (4.407 homens) com faixa etária entre 55-65 anos. Os homens responderam a um questionário desde os 40-50 anos e as mulheres desde os 35-50 anos, período em que também foi monitorado o nível de concentração dos solventes a que eram expostos. $\mathrm{O}$ desempenho neurocognitivo foi avaliado com o Digit Symbol Substitution Test (DSST) e o Mini Exame de Estado Mental (MEEM). As mulheres tiveram desempenho mais elevado que os homens no DSST, mas não houve diferença quanto ao MEEM. A correlação entre o desempenho nos testes e a concentração dos solventes indicou desempenho menor no DSST entre aqueles com alta exposição ao benzeno, categorias de clorados, aromáticos e solventes petrolíferos. Estes dados sugerem que exposições ocupacionais a solventes podem estar associadas com comprometimento cognitivo no envelhecimento.

Com a finalidade de avaliar o desempenho neurocognitivo entre os sexos após alguns meses sem exposição a solventes orgânicos, Morrow e Scott (2002) compararam os resultados de 17 homens e 17 mulheres com exposição crônica (idade entre 31 a 49 anos, escolaridade média de 13 anos e tempo de exposição de 5 anos) no Trail Making Test A, Figuras Complexas de Rey-Osterrieth, e WAIS-R. Estes autores não encontraram diferenças significantes entre os sexos $(p>$ $0,05)$. Entretanto, homens e mulheres demonstraram um padrão diferente de alterações, pois o tempo de exposição afetou significativamente a memória verbal (relacionada a estruturas me- 
dianas ou temporais) dos homens expostos, e a velocidade executiva ou de processamento (relacionada a estruturas mais frontais) das mulheres expostas.

O objetivo do presente estudo foi comparar o desempenho neurocognitivo de homens e mulheres com e sem histórico de exposição crônica a solventes orgânicos e investigar se existem diferenças na memória de curto prazo, atenção, e funções executivas (flexibilidade cognitiva). Especificamente, comparou-se o desempenho entre homens expostos e não expostos; mulheres expostas e não expostas e homens e mulheres expostos. A hipótese principal foi verificar se a exposição crônica aos solventes orgânicos prejudicava o funcionamento neurocognitivo. Caso a hipótese principal fosse aceita, testou-se uma segunda hipótese que foi verificar se os efeitos da exposição crônica aos solventes orgânicos poderiam ser relacionados a alguns índices de composição corpórea (peso, massa gorda, massa corporal magra, relação cintura-quadril, gordura visceral e água corporal) que tendem a se diferenciar entre os sexos.

\section{Método}

\section{Participantes}

Participaram 56 voluntários que formaram quatro grupos: Grupo exposto feminino (GEf), Grupo exposto masculino (GEm), Grupo controle feminino (GCf), Grupo controle masculino (GCm). Os dados sociodemográficos podem ser vistos na Tabela 1 .

Os participantes dos grupos de estudo foram recrutados nos postos de combustíveis, com autorização do Sindicato do Comércio Varejista de Derivados de Petróleo, Brasil. Os participantes dos grupos controle foram recrutados da população em geral, a partir de busca ativa, convidando-se trabalhadores da Universidade sede dos pesquisadores, explicando-se os objetivos e os aspectos éticos do estudo, com os que aceitavam participar eram combinados dia e horário para realização da coleta de dados.

Os participantes do grupo controle foram recrutados por busca ativa. Os grupos foram pare- ados por sexo, idade, e escolaridade na tentativa de eliminar ou reduzir os efeitos destas variáveis nos resultados.

Foram incluídos nos quatro grupos os participantes que possuíam acuidade visual normal ou corrigida (20/20) e aceitaram participar voluntariamente da pesquisa. Adicionalmente, participaram do GE os trabalhadores que exerciam seis meses ou mais de atividade de frentista (Zavalić, Mandić, Turk, Bogadi-Sare, $\&$ Plavec, 1998), nunca usaram equipamentos de proteção individual (EPI's), como máscara ou óculos e exerciam a atividade em turno matutino ou vespertino.

Os critérios de exclusão para os quatro grupos foram: consumo de cigarro, bebida alcoólica e outras drogas, ter sido exposto a vapores químicos em outras profissões, doenças oftalmológicas, neurológicas, psiquiátricas, diabetes ou hipertensão arterial, prática de atividades físicas regulares, todos informados por autorrelato. Foi considerado exercício físico regular a prática de, pelo menos, 30 minutos de atividade com frequência de três ou mais vezes por semana (Bouchard, Blair, \& Haskell, 2012). Exercícios físicos intensos podem aumentar a captação pulmonar de solventes relativamente polares e diminuir o fluxo sanguíneo para o fígado e os rins, diminuindo, assim, a biotransformação dos solventes metabolizados e a eliminação urinária (Klaassen \& Watkins, 2012).

Os participantes dos grupos de estudo trabalhavam em turno matutino ou vespertino com um regime de trabalho em turnos de oito horas/ dia, seis dias/semana, com intervalo de uma hora para almoço. Eles trabalhavam com etanol, gasolina e diesel.

\section{Instrumentos}

Questionário de dados clínicos e sociodemográficos. Teve o objetivo de caracterizar os participantes quanto a idade, escolaridade, consumo de álcool, cigarro ou de outras drogas, doenças oculares, neurológicas, psiquiátricas, diabetes, hipertensão arterial, prática de atividade física, e especificamente para o GE, tempo de exposição, jornada de trabalho por dia e por se- 
Tabela 1

Dados Sociodemográficos referentes a Idade e Escolaridade dos Grupos Expostos e Controle e Tempo de Exposição dos Grupos Expostos (GEm e GEf) em Anos

\begin{tabular}{|c|c|c|c|c|c|c|c|c|c|c|c|c|}
\hline \multirow{2}{*}{ Dados } & \multicolumn{2}{|c|}{$\begin{array}{c}\mathrm{GEm} \\
(n=14)\end{array}$} & \multicolumn{2}{|c|}{$\begin{array}{c}\text { GEf } \\
(n=14)\end{array}$} & \multicolumn{2}{|c|}{$\begin{array}{c}\mathrm{GCm} \\
(n=14)\end{array}$} & \multicolumn{2}{|c|}{$\begin{array}{c}\mathrm{GCf} \\
(n=14)\end{array}$} & \multirow[t]{2}{*}{$\chi^{2}$} & \multirow[t]{2}{*}{$p$} & \multirow[t]{2}{*}{$U$} & \multirow[t]{2}{*}{$p$} \\
\hline & $M$ & $D P$ & $M$ & $D P$ & $M$ & $D P$ & $M$ & $D P$ & & & & \\
\hline Idade & 29,35 & 5,92 & 28 & 7,05 & 27,50 & 7,81 & 26,36 & 6,26 & 3,81 & 0,28 & - & - \\
\hline Escolaridade & 9,71 & 2,05 & 10,07 & 1,21 & 9,73 & 1,85 & 10,50 & 1,09 & 1,42 & 0,70 & - & - \\
\hline Exposição & 5,84 & 4,12 & 4,60 & 3,34 & \multicolumn{2}{|c|}{ Não há } & \multicolumn{2}{|c|}{ Não há } & - & - & 47,5 & 0,31 \\
\hline
\end{tabular}

Nota. $M=$ média; $D P=$ desvio padrão; GEm = Grupo exposto masculino; GEf = Grupo exposto feminino; GCf = Grupo Controle feminino; $\mathrm{GCm}=$ Grupo Controle masculino; $\chi^{2}=$ Teste de Kruskal Wallis; $U=$ Teste $U$ de Mann Whitney.

mana (em horas), uso de EPI's, tipo de material com que trabalhava.

Trail Making Test - TMT (Reitan \& Wolfson, 1985). Este instrumento avalia a atenção, a flexibilidade cognitiva, a procura visual e função motora. $\mathrm{O}$ teste possui duas partes, A e B. A tarefa do participante em ambas as partes é desenhar um trajeto em menor tempo possível sem tirar o lápis do papel. Na Parte A, deve desenhar o trajeto, em ordem crescente, entre os números 1 a $25 . \mathrm{Na}$ Parte $\mathrm{B}$, há uma demanda cognitiva maior, pois deve desenhar o trajeto de 1 a 13 alternando com as letras $\mathrm{A}$ até $\mathrm{L}$, isto é, 1-A, 2-B, 3-C e assim sucessivamente. Dessa forma, o TMT-A foi administrado inicialmente, apresentando ao participante uma folha reduzida, com o objetivo de treiná-lo para executar a tarefa. $\mathrm{O}$ participante foi instruído a ligar os círculos com os números em ordem crescente no menor tempo possível. Em seguida, preencheu a "folha teste" e o tempo foi cronometrado. Ao cometer um erro durante a realização do teste, o voluntário voltava para o círculo anterior e continuava o teste (Reitan, 1958). Dessa maneira, os erros não são registrados, mas aumentam o tempo total (Lezak, 2004). O escore nesta tarefa foi o tempo total em segundos utilizado para ligar os círculos. Já a execução do TMT-B seguiu o mesmo protocolo descrito no TMT-A, mas com agregada tarefa de ligar letras a números de forma alternada, em ordem crescente.

Figura Complexa de Rey - FCR (Oliveira, Rigoni, Andretta, \& Moraes, 2004). Foram utilizadas as fases de Cópia e Reprodução de Memória, que avaliam a codificação e recuperação de memória de curto prazo, respectivamente. Consiste em um cartão com o desenho de uma figura complexa composta por um retângulo grande, bissetores horizontais e verticais, duas diagonais, e detalhes geométricos adicionais interna e externamente ao retângulo grande. São avaliados 18 itens, a pontuação para cada item pode ser 0,1 ou 2 (um para precisão e um para localização), sendo a pontuação máxima de 36 pontos. Na etapa de cópia (FCR-C), o desenho foi apresentado horizontalmente e o participante o copiou em uma folha em branco, sem tempo determinado. Já a etapa Memória (FCR-M) ocorreu três minutos após a cópia. O participante reproduzia a figura sem a presença da cópia ou do modelo. Neste intervalo o participante não realizou nenhuma outra atividade nem foi exposto a estímulos distratores. Os testes neurocognitivos foram corrigidos conforme instruções dos manuais, mas levou-se em consideração a pontuação bruta obtida pelos participantes, pois o objetivo era comparar os grupos expostos a grupos controle com características específicas, o que reduzia os efeitos dos resultados estarem relacionados a erro do Tipo I devido ao não controle de variáveis intervenientes.

Avaliação da composição corporal. A análise de impedância bioelétrica foi realizada através do Inbody 720 (MF-BIA8; Biospace Co. Ltd., Seoul, Korea, 2005). O equipamento possui uma corrente alternada de 250 milliampere (mA) 
e um sistema tetrapolar com oito eletrodos tácteis, utilizando frequências em kilohertz $(\mathrm{kHz})$ de $1 ; 5 ; 50 ; 250 ; 500$ e 1000. Esse equipamento verifica a alteração da impedância em tecidos corporais através do envio de sinais elétricos detectáveis do corpo, sendo gerada a impedância segmentar dos membros superiores e inferiores e tronco para todas as frequências (Gába \& Přidalová, 2014). Os escores de impedância corporal são calculados pela soma dos valores de impedância segmentar. Nesta pesquisa, as medidas tomadas como referência foram: o peso total, o índice de massa corporal magra (MCM), a massa corporal gorda (MCG), Relação Cintura-Quadril (RCQ), área de gordura visceral, água corporal.

\section{Procedimento}

A avaliação neurocognitiva e a avaliação da composição corporal foram realizadas em dias diferentes não excedendo duas semanas entre uma e outra. A avaliação neurocognitiva consistiu na aplicação dos testes TMT-A, TMT-B, FCR Cópia e FCR Memória, sendo realizada no Laboratório de Percepção, Neurociências e Comportamento da Universidade Federal da Paraíba na cidade de João Pessoa-PB. Todos os testes foram administrados por um único profissional da Psicologia que foi treinado durante o período de dois meses por um professor doutor em Psicologia. A duração geral desta avaliação foi em média de 30 minutos.

A avaliação da composição corporal foi realizada por um educador físico no laboratório de Educação Física de uma Instituição de Ensino Superior na cidade de João Pessoa-PB. Os participantes seguiram os critérios recomendados pelo manual do equipamento antes de realizar o exame, por exemplo, não fazer refeição quatro horas antes; reduzir o volume de urina e fezes; permanecer em pé por cinco minutos e, as mulheres, não estar no período menstrual. Esta avaliação durava em média dez minutos. Participaram desta etapa 43 voluntários, os demais justificaram a incompatibilidade de horários ou desistiram.

\section{Aspectos Éticos}

O estudo foi aprovado pelo Comitê de Ética da Universidade Federal da Paraíba com número de Certificado de Apresentação para Apreciação Ética: 21350113.9.0000.5188. A participação ocorreu mediante a assinatura do Termo de Consentimento Livre e Esclarecido, seguindo a Resolução 466/12 do Conselho Nacional de Saúde (2012) que estabelece as diretrizes e normas regulamentadoras de pesquisas envolvendo seres humanos.

\section{Análise dos Dados}

As análises descritivas e inferenciais foram realizadas por meio do software $\mathrm{IBM}{ }^{\circledR}$ SPSS ${ }^{\circledR}$ Statistics versão 20.0.0 (IBM Corporation, Armonk, NY, USA). Adotou-se o critério de significância $p<0,05$. A maioria dos dados não apresentou distribuição normal (KolmogorovSmirnov e Shapiro-Wilk) nem homogeneidade das variâncias (teste de Levene), possivelmente pelo tamanho amostral pequeno, sendo realizada a análise não paramétrica $U$ de Mann-Whitney para comparar o desempenho nos testes entre os grupos: (1) GEf e GCf; (2) GEm e GCm; e (3) GEm e GEf. O tamanho do efeito foi calculado utilizando o coeficiente $r$ de correlação de Pearson que consiste na seguinte fórmula: $r$ $=\mathrm{Z} / \sqrt{ } \mathrm{N}$, onde $Z$ é o escore padronizado e $N$, o número de observações $(n=28$; Dancey, Reidy, \& Rowe, 2017). Os valores limites para o tamanho do efeito foram considerados conforme Cohen (1988) apud Dancey e Reidy (2013), sendo eles, pequeno $(0,20-0,49)$, médio $(0,50$ - 0,79$)$ e grande $(\geq 0,80)$.

A análise $\rho$ de Spearman foi utilizada para correlacionar as medidas de composição corpórea (peso, massa gorda, massa corporal magra, relação cintura-quadril, gordura visceral, água corporal) com os resultados obtidos nos testes neurocognitivos pelos grupos de estudo masculino e feminino. Considerou-se a pontuação bruta obtida nos testes neurocognitivos para a análise dos dados. 


\section{Resultados}

\section{Análises Descritivas}

Testes neurocognitivos. Os escores dos testes TMT-A e TMT-B corresponderam ao tempo de execução da tarefa em segundos, ou seja, quanto menor o tempo para concluir a tarefa, melhor o desempenho. Já as pontuações nos testes FCR-Cópia e FCR-Memória variavam de acordo com a precisão e o bom posicionamento de cada item da figura, ou seja, quanto mais acertos, maior habilidade para codificar e recuperar a informação, respectivamente.

A Tabela 2 apresenta a mediana, primeiro e terceiro quartis e os valores mínimo e máximo obtidos em cada teste pelos grupos GEm, GEf, GCm e GCf. Observa-se na Tabela 2 que, em geral, os grupos expostos (GEm e GEf) apresentaram desempenho menor que os grupos controles (GCm e GCf) nos testes.
Já a Tabela 3 apresenta a mediana, primeiro e terceiro quartis e os valores mínimo e máximo referentes aos índices de composição corporal dos grupos GEm, GEf, GCm e GCf. Em geral, os homens dos grupos exposto e controle apresentaram índices maiores de água corporal, peso, massa gorda, massa magra, RCQ, gordura visceral. Vale ressaltar que a massa de gordura do GEf teve uma variação mínima e máxima superior $($ Mínimo $=11,60 ;$ Máximo $=43,60)$ ao GEm $($ Mínimo =7,50; Máximo =33,20).

\section{Análises Inferenciais}

Testes neurocognitivos. Foram realizadas comparações dos postos (Teste $U$ de Mann-Whitney) entre a mediana dos grupos: (1) GEf e GCf; (2) GEm e GCm; (3) GEf e GEm, conforme pode ser observado na Tabela 4.

O desempenho do GEf em comparação ao GCf foi significativamente menor em todos os testes, com magnitude do efeito grande. $\mathrm{O}$

Tabela 2

Estatística Descritiva dos Postos da Mediana, Valores do Primeiro e Terceiro Quartis, Mínimo e Máximo Obtidos nos Testes TMT-A, TMT-B, FCR - C e FCR - M dos Grupos Expostos (GEf, GEm) e Não Expostos (GCm e GCf)

\begin{tabular}{|c|c|c|c|c|c|c|c|c|c|c|}
\hline \multirow{2}{*}{$\begin{array}{c}\text { Testes } \\
\text { neurocognitivos }\end{array}$} & \multicolumn{5}{|c|}{$\operatorname{GEm}(n=14)$} & \multicolumn{5}{|c|}{$\operatorname{GEf}(n=14)$} \\
\hline & $M d n$ & $Q_{1}$ & $Q_{3}$ & Mín. & Máx. & $M d n$ & $Q_{1}$ & $Q_{3}$ & Mín. & Máx. \\
\hline TMT - A & 37,50 & 30,75 & 44,25 & 23,00 & 85,00 & 40,50 & 22,75 & 47,50 & 21,00 & 55,00 \\
\hline TMT - B & 77,50 & 54,25 & 107,00 & 36,00 & 163,00 & 85,50 & 50,50 & 73,50 & 45,00 & 177,00 \\
\hline FCR - C & 34,00 & 29,87 & 36,00 & 28,00 & 36,00 & 32,25 & 35,75 & 36,00 & 20,50 & 36,00 \\
\hline FCR- M & 20,25 & 14,25 & 23,75 & 4,00 & 33,00 & 17,50 & 24,37 & 30,50 & 8,50 & 27,00 \\
\hline \multirow{2}{*}{$\begin{array}{c}\text { Testes } \\
\text { neurocognitivos }\end{array}$} & \multicolumn{5}{|c|}{$\operatorname{GCm}(n=14)$} & \multicolumn{5}{|c|}{$\operatorname{GCf}(n=14)$} \\
\hline & $M d n$ & $Q_{1}$ & $Q_{3}$ & Mín. & Máx. & Mdn & $Q_{1}$ & $Q_{3}$ & Mín. & Máx. \\
\hline TMT - A & 25,00 & 26,50 & 47,50 & 17,00 & 54,00 & 29,50 & 24,50 & 34,50 & 18,00 & 45,00 \\
\hline TMT - B & 68,50 & 62,50 & 99,75 & 40,00 & 92,00 & 58,00 & 44,75 & 63,75 & 36,00 & 100,00 \\
\hline FCR - C & 36,00 & 35,75 & 36,00 & 34,50 & 36,00 & 36,00 & 36,00 & 36,00 & 34,00 & 36,00 \\
\hline FCR - M & 30,00 & 12,75 & 22,25 & 21,00 & 33,00 & 36,00 & 28,50 & 34,00 & 18,00 & 36,00 \\
\hline
\end{tabular}

Nota. TMT-A (Trail Making Test A), TMT-B (Trail Making Test B), FCR - C (Figura Complexa de Rey - Cópia), FCR M (Figura Complexa de Rey - Memória), GEm (grupo exposto masculino), GEf (grupo exposto feminino), GCm (grupo controle masculino), GCf (grupo controle feminino), Mdn (mediana), $n$ (número de participantes), $Q_{1}\left(1^{\circ}\right.$ Quartil), $Q_{3}\left(3^{\circ}\right.$ Quartil), Mín. (Mínimo), Máx. (Máximo). A mediana (ou percentil 50) é o valor que divide os dados ordenados ao meio. Os quartis inferior $\left(1^{\circ}\right.$ Quartil) e superior $\left(3^{\circ}\right.$ Quartil) são os valores abaixo dos quais estão um quarto e três quartos dos dados, respectivamente. 
Tabela 3

Estatística Descritiva dos Postos da Mediana, Valores do Primeiro e Terceiro Quartis, Mínimo e Máximo dos Índices de Composição Corporal dos Grupos Expostos (GEf, GEm) e Não Expostos (GCm e GCf)

\begin{tabular}{|c|c|c|c|c|c|c|c|c|c|c|}
\hline \multirow{2}{*}{ Composição corporal } & \multicolumn{5}{|c|}{$\operatorname{GEm}(n=11)$} & \multicolumn{5}{|c|}{$\operatorname{GEf}(n=10)$} \\
\hline & $M d n$ & $Q 1$ & $Q 3$ & Min. & Máx. & $M d n$ & $Q 1$ & $Q 3$ & Min. & Máx. \\
\hline Água corporal $(l)$ & 37,90 & 36,70 & 48,80 & 33,10 & 52,90 & 30,70 & 28,90 & 32,30 & 25,40 & 36,30 \\
\hline Peso (Kg) & 73,50 & 67,90 & 90,70 & 52,60 & 103,80 & 58,60 & 54,30 & 66,70 & 50,80 & 88,90 \\
\hline Massa gorda (Kg) & 23,70 & 10,90 & 28,00 & 7,50 & 33,20 & 18,30 & 15,60 & 22,50 & 11,60 & 43,60 \\
\hline Massa magra (Kg) & 48,80 & 47,30 & 62,90 & 42,60 & 67,90 & 39,60 & 37,10 & 41,50 & 32,60 & 46,70 \\
\hline $\mathrm{RCQ}(\mathrm{cm})$ & 0,93 & 0,87 & 0,97 & 0,70 & 1,03 & 0,88 & 0,86 & 0,95 & 0,83 & 1,13 \\
\hline Gordura visceral $\left(\mathrm{cm}^{2}\right)$ & 114,00 & 61,50 & 118,80 & 29,20 & 151,80 & 90,90 & 73,90 & 97,30 & 57,60 & 196,60 \\
\hline \multirow{2}{*}{ Composição corporal } & \multicolumn{5}{|c|}{$\operatorname{GCm}(n=11)$} & \multicolumn{5}{|c|}{$\operatorname{GCf}(n=11)$} \\
\hline & $M d n$ & $Q 1$ & $Q 3$ & Min. & Máx. & $M d n$ & $Q 1$ & $Q 3$ & Min. & Máx. \\
\hline Água corporal (1) & 41,90 & 37,30 & 48,30 & 31,3 & 53 & 28,40 & 27,20 & 34,50 & 24,7 & 40,8 \\
\hline Peso (Kg) & 81,80 & 60,10 & 84,30 & 48,10 & 123,10 & 66,00 & 55,00 & 75,80 & 48,70 & 102,50 \\
\hline Massa gorda (Kg) & 18,50 & 11,30 & 26,80 & 5,40 & 50,90 & 24,00 & 21,10 & 33,30 & 12,40 & 48,00 \\
\hline Massa magra (Kg) & 54,20 & 48,10 & 62,20 & 40,40 & 68,00 & 36,40 & 34,90 & 41,40 & 31,70 & 51,30 \\
\hline $\mathrm{RCQ}(\mathrm{cm})$ & 0,90 & 0,86 & 0,97 & 0,80 & 1,04 & 0,90 & 0,86 & 0,98 & 0,81 & 0,99 \\
\hline Gordura visceral $\left(\mathrm{cm}^{2}\right)$ & 80,30 & 56,50 & 121,20 & 18,60 & 204,6 & 115,90 & 100,00 & 142,00 & 59,40 & 172,10 \\
\hline
\end{tabular}

Nota. $n$ (número de participantes), $M d n$ (mediana), $D P$ (desvio padrão), GEm (grupo exposto masculino), GEf (grupo exposto feminino), GCm (grupo controle masculino), GCf (grupo controle feminino), 1 (litro), $\mathrm{Kg}$ (quilograma), cm (centímetros), $\mathrm{cm}^{2}$ (centímetro quadrado).

desempenho do GEm em comparação com o $\mathrm{GCm}$ também foi significantemente menor em todos os testes, exceto no TMT-B $(p>0,05)$. No TMT-A a magnitude de efeito foi média ( $U=$ $44,50 ; p=0,01 ; r=0,47)$, no FCR-Cópia $(U=$ $46,50 ; p=0,009 ; r=0,50)$ e no FCR-Memória $(U=29,00 ; p=0,001 ; r=0,60)$ a magnitude de efeito foi grande.

O GEf em comparação ao GEm levou mais tempo para realizar o TMT - A e TMT - B e acertaram menos elementos da FCR nas etapas de codificação e memória, apesar disto não houve diferença significativa entre os grupos em nenhum dos testes.

\section{Composição Corporal}

A análise da composição corporal mostrou que as mulheres expostas apresentaram nível de água corporal menor em comparação aos homens expostos $(U=3,0 ; p<0,001)$. O grupo de mulheres expostas também apresentou menos massa magra corporal comparado ao grupo de homens expostos $(U=3,0 ; p<0,001)$. Os dados são apresentados na Tabela 5.

\section{Análises Correlacionais}

Testou-se a hipótese secundária de que o desempenho de homens e mulheres poderia ser influenciado por medidas de composição corporal. Assim, foram realizadas análises correlacionais (Coeficiente Correlacional de Spearman) entre o desempenho nos testes neurocognitivos e as medidas de água corporal, peso, massa magra, massa gorda, RCQ e gordura visceral, como é possível observar na Tabela 6 . 
Tabela 4

Comparação entre os Escores dos Grupos de Estudo e Grupos Controle, Apresentando a Estatística $U$ de Mann Whitney, Nível de Significância e Tamanho do Efeito para Cada Teste

\begin{tabular}{lcccccccccc}
\hline & \multicolumn{4}{c}{ GEf x GCf } & \multicolumn{2}{c}{ GEm x GCm } & \multicolumn{4}{c}{ GEf x GEm } \\
\cline { 2 - 11 } Testes neurocognitivos & $U$ & $p$ & $r$ & $U$ & $p$ & $r$ & $U$ & $p$ & $r$ \\
\hline TMT - A & 48,50 & $0,02^{*}$ & 0,43 & 44,50 & $0,01 *$ & 0,47 & 89,00 & 0,68 & 0,08 \\
TMT - B & 39,50 & $0,007 * *$ & 0,51 & 65,50 & 0,13 & 0,28 & 90,50 & 0,73 & 0,07 \\
FCR - C & 16,00 & $0,001 * *$ & 0,76 & 46,50 & $0,009 * *$ & 0,50 & 79,00 & 0,38 & 0,19 \\
FCR- M & 16,5 & $0,001 * *$ & 0,71 & 29,00 & $0,001 * *$ & 0,60 & 87,50 & 0,63 & 0,09 \\
\hline
\end{tabular}

Nota. TMT-A (Trail Making Test A), TMT-B (Trail Making Test B), FCR - C (Figura Complexa de Rey - Cópia), FCR - M (Figura Complexa de Rey - Memória), GEm (grupo exposto masculino), GEf (grupo exposto feminino), GCm (grupo controle masculino), GCf (grupo controle feminino); $U$ (Estatística de Mann Whitney), $r$ (tamanho do efeito).

$* p<0,05$. ${ }^{* *} p<0,01$.

Tabela 5

Comparação entre os Escores dos Índices de Composição Corporal dos Grupos de Estudo e Grupos Controle, Apresentando a Estatística U de Mann Whitney, Nível de Significância

\begin{tabular}{lcccccc}
\hline \multirow{2}{*}{ Composição corporal } & \multicolumn{2}{c}{ GEf x GCf } & GEm x GCm & \multicolumn{2}{c}{ GEf x GEm } \\
\cline { 2 - 6 } & $U$ & $p$ & $U$ & $p$ & $U$ & $p$ \\
\hline Água corporal (1) & 54,50 & 0,69 & 55,00 & 0,72 & 3,00 & $0,001^{*}$ \\
Peso (Kg) & 49,00 & 0,45 & 56,00 & 0,76 & 54,00 & 0,01 \\
Massa gorda (Kg) & 38,00 & 0,13 & 53,50 & 0,65 & 58,00 & 0,87 \\
Massa magra (Kg) & 48,00 & 0,41 & 54,50 & 0,69 & 3,00 & $0,001 *$ \\
RCQ (cm) & 48,00 & 0,41 & 59,00 & 0,92 & 50,50 & 0,51 \\
Gordura visceral $\left(\mathrm{cm}^{2}\right)$ & 32,00 & 0,06 & 52,00 & 0,58 & 58,00 & 0,87 \\
\hline
\end{tabular}

Nota. TMT-A (Trail Making Test A), TMT-B (Trail Making Test B), FCR - C (Figura Complexa de Rey - Cópia), FCR - M (Figura Complexa de Rey - Memória), GEm (grupo exposto masculino), GEf (grupo exposto feminino), GCm (grupo controle masculino), GCf (grupo controle feminino); $U$ (Estatística de Mann Whitney), 1 (litro), $\mathrm{Kg}$ (quilograma), cm (centímetros), cm2 (centímetro quadrado).

$* p<0,001$.

Observa-se na Tabela 6 que os resultados das correlações do Grupo exposto feminino indicaram correlação positiva significativa entre o escore no teste FCR-Cópia e as medidas de água $(\mathrm{CC}=0,77 ; p=0,005)$, peso $(\mathrm{CC}=-0,86$; $p=0,001)$, massa gorda $(\mathrm{CC}=0,70 ; p=0,02) \mathrm{e}$ massa magra $(\mathrm{CC}=0,76 ; p=0,007)$.

Em relação ao Grupo exposto masculino, os resultados mostraram correlação positiva e significativa entre os escores no TMT - B com as medidas de RCQ $(\mathrm{CC}=0,73 ; p=0,01)$ e gordura visceral $(\mathrm{CC}=0,66 ; p=0,03)$.

\section{Discussão}

O objetivo deste estudo foi verificar o desempenho neurocognitivo de homens e mulheres com e sem histórico de exposição crônica a solventes orgânicos. A hipótese principal foi investigar se a exposição prejudicaria o desempenho neurocognitivo. Os principais resultados encon- 
Tabela 6

Relação entre as Medidas de Composição Corpórea com os Escores nos Testes Neurocognitivos do Grupo Exposto Feminino (GEf) e Grupo Exposto Masculino (GEm)

\begin{tabular}{|c|c|c|c|c|c|c|c|c|}
\hline \multirow{3}{*}{ Composição corporal } & \multicolumn{8}{|c|}{ GEf } \\
\hline & \multicolumn{2}{|c|}{ TMT - A } & \multicolumn{2}{|c|}{ TMT - B } & \multicolumn{2}{|c|}{ FCR - C } & \multicolumn{2}{|c|}{ FCR - M } \\
\hline & $\mathrm{CC}$ & $p$ & $\mathrm{CC}$ & $p$ & $\mathrm{CC}$ & $p$ & $\mathrm{CC}$ & $p$ \\
\hline Água corporal $(l)$ & $-0,29$ & 0,38 & $-0,11$ & 0,73 & 0,77 & $0,005 * *$ & $-0,06$ & 0,86 \\
\hline Peso (Kg) & $-0,22$ & 0,50 & 0,15 & 0,65 & 0,86 & $0,001 * *$ & 0,16 & 0,63 \\
\hline Massa gorda (Kg) & $-0,13$ & 0,69 & 0,44 & 0,18 & 0,70 & $0,02 *$ & 0,24 & 0,48 \\
\hline Massa magra (Kg) & $-0,27$ & 0,42 & $-0,09$ & 0,79 & 0,76 & $0,007 * *$ & $-0,09$ & 0,80 \\
\hline $\mathrm{RCQ}(\mathrm{cm})$ & $-0,23$ & 0,50 & $-0,02$ & 0,96 & 0,44 & 0,17 & 0,05 & 0,88 \\
\hline \multirow[t]{3}{*}{ Gordura visceral $\left(\mathrm{cm}^{2}\right)$} & 0,03 & 0,94 & 0,46 & 0,16 & 0,58 & 0,06 & 0,18 & 0,59 \\
\hline & \multicolumn{8}{|c|}{ GEm } \\
\hline & \multicolumn{2}{|c|}{ TMT - A } & \multicolumn{2}{|c|}{ TMT - B } & \multicolumn{2}{|c|}{ FCR - C } & \multicolumn{2}{|c|}{ FCR - M } \\
\hline Composiçao corporal & $\mathrm{CC}$ & $p$ & $\mathrm{CC}$ & $p$ & $\mathrm{CC}$ & $p$ & $\mathrm{CC}$ & $p$ \\
\hline Água corporal $(l)$ & $-0,47$ & 0,15 & $-0,16$ & 0,65 & 0,34 & 0,31 & 0,03 & 0,94 \\
\hline Peso (Kg) & $-0,31$ & 0,28 & $-0,15$ & 0,62 & 0,13 & 0,66 & 0,11 & 0,71 \\
\hline Massa gorda (Kg) & $-0,14$ & 0,69 & 0,52 & 0,10 & $-0,45$ & 0,16 & $-0,42$ & 0,20 \\
\hline Massa magra (Kg) & $-0,48$ & 0,14 & $-0,15$ & 0,67 & 0,29 & 0,39 & $-0,01$ & 0,99 \\
\hline $\mathrm{RCQ}(\mathrm{cm})$ & 0,08 & 0,81 & 0,73 & $0,01^{*}$ & $-0,58$ & 0,06 & $-0,55$ & 0,08 \\
\hline Gordura visceral $\left(\mathrm{cm}^{2}\right)$ & $-0,02$ & 0,95 & 0,66 & $0,03^{*}$ & $-0,52$ & 0,10 & $-0,49$ & 0,12 \\
\hline
\end{tabular}

Nota. TMT-A (Trail Making Test A), TMT-B (Trail Making Test B), FCR - C (Figura Complexa de Rey - Cópia), FCR - M (Figura Complexa de Rey - Memória), $\rho$ (Coeficiente de correlação de postos de Spearman), 1 (litro), Kg (quilograma), cm (centímetros), $\mathrm{cm}^{2}$ (centímetro quadrado). TMT (Trail Making Test), FCR (Figura Complexa de Rey); CC = coeficiente de correlação. $* p<0,05 ; * * p<0,01$

trados confirmam a hipótese principal, pois a exposição crônica aos solventes orgânicos alterou o desempenho neurocognitivo de mulheres e homens expostos em comparação aos grupos controle.

Os resultados mostraram que as mulheres expostas em comparação às mulheres não expostas obtiveram desempenho significantemente menor em todas as tarefas avaliadas pelos testes TMT-A, TMT-B, FCR-Cópia e FCR-Memória. Estes resultados demonstram a possível influência da exposição aos solventes sobre o funcionamento neurocognitivo das mulheres.
Estes dados corroboram o estudo de LoSasso, Rapport, Axelrod e Whitman (2002) que identificou que trabalhadoras de salão de beleza [expostas a solventes orgânicos e (Meth)Acrylates] em comparação ao grupo controle, tiveram escores mais baixos nas medidas de atenção e flexibilidade cognitiva (TMT-B). Ainda confirma os achados de Morrow, Robin, Hodgson e Kamis (2002) que sugerem que pessoas expostas a substâncias neurotóxicas geralmente apresentam declínio na memória de curto prazo.

No entanto, este resultado difere dos achados de Parkinson et al. (1990) com mulheres sob 
diferentes graus de exposição a solventes orgâni$\cos (n=494)$ comparadas a mulheres não expostas $(n=73)$, já que os autores não encontraram diferenças nos domínios neurocognitivos (funcionamento visuoperceptual; atenção/integração psicomotora e velocidade motora/destreza manual). Não é possível fazer uma comparação direta entre o nosso estudo e o de Parkinson et al. (1990), pois existem algumas diferenças básicas. Por exemplo, eles avaliaram mulheres com categorias diferentes de exposição aos solventes orgânicos, enquanto o presente estudo foi realizado com mulheres com mais de seis meses de exposição

Contudo, o resultado do presente estudo era esperado, pois o sistema nervoso central (SNC) é um dos órgãos mais vulneráveis aos efeitos dos solventes, tendo em vista que possui grande concentração lipídica (Fueta, Fukuda, Ishidao, \& Hori, 2004). Além disso, os dados corroboram estudo que aponta os efeitos cumulativos da exposição crônica (Zavalić et al., 1998).

Os homens expostos em comparação aos homens não expostos apresentaram desempenho significantemente menor nos testes TMT-A, FCR-Cópia e FCR-Memória, exceto no TMT-B (Tabelas $1 \mathrm{e} 2$ ). Este resultado era esperado, pois achados semelhantes foram encontrados por outros estudos realizados com homens (Saygun et al., 2012; Song et al., 2015; Thetkathuek et al., 2015).

Estes estudos têm identificado que a memória de curto prazo relacionada a informações verbais, visuais e visuoespaciais é uma das funções neurocognitivas mais sensíveis aos efeitos dos solventes. O que tem sido comprovado inclusive por técnicas de imageamento cerebral que mostraram ativação menor em regiões ligadas a memória, como o corpo estriado, córtex cingulado anterior, préfrontal e parietal em indivíduos com exposição crônica a solventes orgânicos (Tang et al., 2011; Visser et al., 2008).

No presente estudo não foi encontrada diferença significante em relação à flexibilidade cognitiva do grupo de homens expostos em comparação ao grupo não exposto. Isto pode ser justificado em parte porque segundo Lotti et al. (2015), os comprometimentos cognitivos referentes a efeitos neurotóxicos não estão bem caracterizados, inclusive alguns estudos não têm encontrado diferenças. Por exemplo, Saygun et al. (2012) não identificaram diferenças no desempenho entre homens expostos e não expostos em relação a orientação espacial, memória de curto prazo, atenção e linguagem, avaliados com o Mini Exame do Estado Mental.

As divergências entre os estudos podem estar relacionadas a diferenças metodológicas, como o tipo de medida utilizada, condições de administração dos testes, tipo de tratamento estatístico aplicado aos dados. As divergências podem ainda estar relacionadas a diferenças nos parâmetros de exposição, tais como a duração (e.g. crônica, subcrônica, aguda), tipo de agentes tóxicos, frequência e nível de concentração (Kim, Jahan, Kabir, \& Brown, 2013); além da forma como o agente é metabolizado e a suscetibilidade global do sistema biológico do indivíduo (Klaassen \& Watkins, 2012). O que dificulta o estabelecimento da relação entre exposição tóxica e alterações neurocognitivas (Wood \& Liossi, 2005). Não obstante, os resultados aqui apresentados sinalizam a possibilidade de os solventes alterarem as estruturas cerebrais relacionadas a atenção, a codificação da informação e a memória de curto prazo dos homens.

Os resultados não mostraram diferenças no desempenho das mulheres expostas em comparação aos homens expostos nos testes TMT-A, TMT-B, FCR-Cópia e FCR-Memória (Tabela 2). Deste modo, não foi possível verificar diferenças no desempenho neurocognitivo relacionadas ao sexo.

Este resultado corrobora o estudo de Morrow e Scott (2002) que não encontrou diferenças entre homens e mulheres expostos nos testes TMT-A, TMT-B, e FCR. Entretanto, Morrow e Scott (2002) não utilizaram grupo controle como parâmetro para verificar a proporção dos efeitos. É possível que o estudo de Morrow e Scott (2002) não tenha encontrado 
diferenças nos resultados entre homens e mulheres por que os efeitos dos solventes já houvessem sido dissipados, pois o tempo médio entre a exposição e a avaliação foi de 10 meses para homens e 9 meses para mulheres, enquanto no presente estudo os frentistas estavam no exercício da profissão.

Já a hipótese secundária foi investigar a relação entre os resultados de homens e mulheres expostos nos testes neurocognitivos com os índices de composição corpórea. Esperava-se que os efeitos da exposição crônica aos solventes prejudicassem o desempenho neurocognitivo e que os efeitos fossem mais acentuados nos homens do que nas mulheres, por causa das diferenças na composição corporal (Gochfeld, 2007; Mergler, 2012; Tomicic \& Vernez, 2014; Vahter et al., 2007; Weiss, 2012). Isto é, como os solventes orgânicos são compostos lipofílicos e as mulheres, geralmente, apresentam concentração de gordura maior, era esperado que o tecido adiposo funcionasse como o fator de proteção nas mulheres (Klaassen \& Watkins, 2012).

É interessante destacar que os resultados das correlações mostraram um padrão diferente de alterações relacionadas com a composição corpórea. Em relação a composição corporal feminina, apenas encontrou-se correlação entre o desempenho no teste FCR-Cópia que avalia o processo de codificação da memória com os índices de água, peso, massa gorda, massa magra. Enquanto que no grupo de homens expostos encontrou-se correlação significativa apenas com o desempenho em flexibilidade mental (TMT - B) e as medidas de RCQ e gordura visceral. Em conclusão os resultados das mulheres pode indicar que com o aumento do peso, massa gorda, massa magra e gordura visceral, maior a tendência à simplificação do desenho, ou seja, observam e codificam poucos detalhes de uma figura complexa, o que estaria relacionado a uma possível alteração no hemisfério esquerdo. Desta forma, ao contrário do que se esperava, o tecido adiposo não funcionou como o fator de proteção nas mulheres, possivelmente porque as mulheres apresentaram menos tecido adiposo em comparação aos homens.

Este resultado pode ser explicado com base nos estudos de Lezak (2004) que identificou que pacientes com lesões no hemisfério esquerdo tendem a manter a memória da estrutura global da figura preservada, porém com perda de detalhes e simplificação do desenho. Já pacientes com lesões no hemisfério direito tendem a perder aspectos gerais do desenho. O estudo mostrou ainda que pessoas que fazem uma cópia incorreta baseada mais em uma baixa organização dos dados do que em uma desordem na habilidade visuoespacial (mais comum com lesões no hemisfério esquerdo), podem melhorar sua performance na tarefa de memória imediata.

Em relação aos homens expostos, o resultado corrobora parcialmente a hipótese de que os solventes podem ter um volume relativamente maior de distribuição e serem metabolizados mais lentamente nas mulheres em comparação aos homens (Gandhi et al., 2004). Isto é, os índices mais elevados de RCQ e gordura visceral podem interferir na forma como os solventes distribuem-se e exercem seus efeitos nos homens.

É importante destacar que ambos os grupos apresentavam idade, escolaridade, hábitos de vida semelhantes, o que aumenta a probabilidade de os resultados serem provenientes do efeito dos solventes.

A literatura tem discutido que as alterações neurocognitivas relacionadas à exposição aos solventes orgânicos podem estar relacionadas aos polimorfismos genéticos nas enzimas glutathione S-transferase theta 1 (GSTT1) and glutathione S-transferase mu 1 (GSTM 1; Palma, Mozzoni, Mutti, Calzetti, \& Negrotti, 1998), ou a diminuição do fator neurotrófico Serum B-cell lymphoma 2 ( $B c l$-2), derivado do cérebro, vulnerável a uma variedade de efeitos tóxicos e envolvido nas funções cognitivas (MonatDescamps \& Deschamps, 2012).

\section{Considerações Finais}

Em conclusão, este estudo aponta que homens e mulheres podem ser atingidos pelos sol- 
ventes, mas a forma como os solventes interagem com o organismo de homens e de mulheres varia. Nas mulheres, a memória pode ser mais prejudicada em função da quantidade de água corporal, peso, massa gorda, massa magra, já nos homens a flexibilidade cognitiva pode ser a mais deteriorada em função dos elevados índices de RCQ e gordura visceral. Portanto, considera-se que a ação dos solventes pode estar mais relacionada aos índices de composição corporal do que ao sexo.

Contudo, trata-se de um estudo inicial e novas pesquisas precisam ser conduzidas para esclarecer o mecanismo pelo qual a composição corpórea pode interferir na absorção dos solventes orgânicos e alterar o funcionamento neurocognitivo, uma vez que este estudou apresentou limitações. Por exemplo, $n$ amostral pequeno, utilizou-se apenas correlações, o que não permitiu controlar variáveis sociodemográficas e tempo de exposição.

Deste modo, sugere-se a realização de estudos longitudinais e experimentais com modelos animais, por exemplo, para verificar os efeitos em outros domínios cognitivos e comparar a magnitude destes efeitos entre o sexo masculino e feminino, considerando de forma mais sistemática a composição corpórea, difícil de padronizar em humanos.

Estes resultados apresentam implicações científicas e sociais importantes. Eles sustentam a importância de avaliar as funções neurocognitivas precocemente, pois alterações nestas funções podem prejudicar o desempenho no trabalho e em diversas atividades da vida cotidiana. Desta maneira, podem ser tornar preditoras de complicações mais graves, como encefalopatia neurotóxica crônica e transtornos neurodegenerativos.

\section{Referências}

Barbosa, I. A. J., Boon, M. Y., \& Khuu, S. K. (2015). Exposure to organic solvents used in dry cleaning reduces low and high level visual function. PLoS ONE, 10(5), e0121422. doi: 10.1371/journal.pone.0121422
Beasley, T. E., Evansky, P. A., \& Bushnell, P. J. (2012). Behavioral effects of sub-acute inhalation of toluene in adult rats. Neurotoxicology and Teratology, 34(1), 83-89. doi: 10.1016/j. ntt.2011.11.001

Berenguer, P., Soulage, C., Perrin, D., Pequignot, J.M., \& Abraini, J. H. (2003). Behavioral and neurochemical effects induced by subchronic exposure to $40 \mathrm{ppm}$ toluene in rats. Pharmacology Biochemistry and Behavior, 74(4), 997-1003. doi: 10.1016/S0091-3057(03)00027-3

Berr, C., Vercambre, M. N., Bonenfant, S., Manoux, A. S., Zins, M., \& Goldberg, M. (2010). Occupational exposure to solvents and cognitive performance in the GAZEL cohort: Preliminary results. Dementia and Geriatric Cognitive Disorders, 30(1), 12-19. doi: 10.1159/000315498

Biospace. (2005). InBody 720 User's Manual. Retrieved from http://www.imr-switzerland.org/ downloads/in-body-720-manual.pdf

Blazer, D. G., Yaffe, K., \& Liverman, C. T. (2015). Characterizing and assessing cognitive aging. Washington, DC: National Academies Press. Retrieved from https://www.ncbi.nlm.nih.gov/ books/NBK316201/

Bouchard, B., Blair, S. N., \& Haskell, W. (2012). Physical Activity and Health (2 ${ }^{\text {nd }}$ Ed.). Champaign, IL: Human Kinetics. Retrieved from http:/www.humankinetics.com/products/allproducts/physical-activity-and-health-2nd-edition

Conselho Nacional de Saúde. (2012). Resolução $n^{o}$ 466, de 12 de dezembro de 2012. Dispõe sobre diretrizes e normas regulamentadoras de pesquisas envolvendo seres humanos. Recuperado em http://conselho.saude.gov.br/resolucoes/2012/ Reso466.pdf

Dancey, C. P., \& Reidy, J. (2013). Estatística sem Matemática para Psicologia (5. ed.). Porto Alegre, RS: Artmed.

Dancey, C., Reidy, J., \& Rowe, R. (2017). Estatística sem matemática para as ciências da saúde. Porto Alegre, RS: Penso.

Ernstgård, L., Gullstrand, E., Löf, A., \& Johanson, G. (2002). Are women more sensitive than men to 2-propanol and m-xylene vapours? Occupational and Environmental Medicine, 59, 759-767. doi:10.1136/oem.59.11.759 
Fueta, Y., Fukuda, T., Ishidao, T., \& Hori, H. (2004). Electrophysiology and immunohistochemistry in the hippocampal cal and the dentate gyrus of rats chronically exposed to 1-bromopropane, a substitute for specific chlorofluorocarbons. Neuroscience, 124(3), 593-603. doi: 10.1016/j.neuroscience.2003.12.025

Gába, A., \& Přidalová, M. (2014). Age-related changes in body composition in a sample of Czech women aged 18-89 years: A cross-sectional study. European Journal of Nutrition, 53(1), 167-176. doi: 10.1007/s00394-013-0514-x

Gandhi, M., Aweeka, F., Greenblatt, R. M., \& Blaschke, T. F. (2004). Sex differences in pharmacokinetics and pharmacodynamics. Annual Review of Pharmacology and Toxicology, 44(1), 499-523. doi: 10.1146/annurev.pharmtox.44.101802.121453

Gleiter, C. H., \& Gundert-Remy, U. (1996). Gender differences in pharmacokinetics. European Journal of Drug Metabolism and Pharmacokinetics, 21(2), 123-128. doi: doi:10.1007/BF03190260

Gochfeld, M. (2007). Framework for gender differences in human and animal toxicology. Environmental Research, 104, 4-21. doi: 10.1016/j.envres.2005.12.005

International Labour Office. (2014, August 25). Safety and health at work: A vision for sustainable prevention. In $X X$ World Congress on Safety and Health at Work 2014: Global Forum for Prevention. Frankfurt, Germany: Author. Retrieved from http://www.ilo. org/wcmsp5/groups/public/@ed_protect/@ protrav/@safework/documents/publication/ wcms_301214.pdf

Kawai, T., Takeuchi, A., \& Ikeda, M. (2015). Comparison of the exposure-excretion relationship between men and women exposed to organic solvents. Journal of Occupational Health, 57(3), 302-305. doi: 10.1539/joh.14-0240-OA

Kim, K.-H., Jahan, S. A., Kabir, E., \& Brown, R. J. C. (2013). A review of airborne polycyclic aromatic hydrocarbons (PAHs) and their human health effects. Environment International, 60, 71-80. doi: 10.1016/j.envint.2013.07.019

Klaassen, C. D., \& Watkins, J. B., III. (2012). Fundamentos em toxicologia de Casarrett e Doull. Porto Alegre, RS: Artmed.
Lezak, M. D. (2004). Neuropsychological Assessment $\left(4^{\text {th }}\right.$ Ed.). New York: Oxford University Press.

Lof, A., \& Johanson, G. (1998). Toxicokinetics of organic solvents: A review of modifying factors. Critical Reviews in Toxicology, 28, 571-650. doi: 10.1080/10408449891344272

LoSasso, G. L, Rapport, L. J., Axelrod, B. N., \& Whitman, R. D. (2002). Neurocognitive sequelae of exposure to organic solvents and (meth)acrylates among nail-studio technicians. Neuropsychiatry Neuropsychology Behavior Neurology, 15(1), 44-55.

Lotti, M., Bleecker, M. L., \& Aminoff, M. J. (2015). Occupational neurology. New York: Elsevier.

Malek, F. A., Möritz, K.-U., \& Fanghänel, J. (2003). A study on specific behavioral effects of formaldehyde in the rat. Journal of Experimental Animal Science, 3(42), 160-170. doi: 10.1016/ S0939-8600(03)80009-3

Mergler, D. (2012). Neurotoxic exposures and effects: Gender and sex matter! Hänninen Lecture 2011. NeuroToxicology, 33(4), 644651. doi: 10.1016/j.neuro.2012.05.009

Monat-Descamps, C., \& Deschamps, F. (2012). Nervous system disorders induced by occupational and environmental toxic exposure. Open Journal of Preventive Medicine, 2(3), 272-278. doi:10.4236/ojpm.2012.23039

Morrow, L. A., \& Scott, A. (2002). Comparison of neuropsychological test scores between men and women with prior exposure to organic solvents. Applied neuropsychology, 9(4), 240-243. doi: 10.1207/S15324826AN0904 7

Morrow, L. A., Robin, N., Hodgson, M. K., \& Kamis, H. (1992). Assessment of attention and memory efficiency in persons with solvent neurotoxicity. Neuropsychologia, 30, 911-922. doi: 10.1016/0028-3932(92)90035-K

Occupational Safety and Health Service. (1998). Chronic organic solvent neurotoxicity. Diagnostic criteria. In Occupational Safety and Health Service, Occupational Safety and Health Information Series, p. 1-15. II. New Zealand: Author.

Oliveira, M., Rigoni, M., Andretta, I., \& Moraes, J. F. (2004). Validação do Teste Figuras Complexas de Rey na população brasileira. Avalia- 
ção Psicológica, 3(1), 33-38. Recuperado em http://pepsic.bvsalud.org/scielo.php?script=sci arttext\&pid=S1677-04712004000100004\&lng= $\mathrm{pt \& t} \operatorname{lng}=\mathrm{pt}$

Palma, G., Mozzoni, P., Mutti, A., Calzetti, S., \& Negrotti, A. (1998). Case-control study of interactions between genetic and environmental factors in Parkinson's disease. Lancet, 352, 1986-1987. doi: 10.1016/S0140-6736(05)61332-3

Parkinson, D. K., Bromet, E. J., Cohen, S., Dunn, L. O., Dew, M. A., Ryan, C., \& Schwartz, J. E. (1990). Health effects of long-term solvent exposure among women in blue-collar occupations. American Journal of Industrial Medicine, 17(6), 661-675. doi: 10.1002/ajim.4700170602

Reitan, R. (1958). Validity of the Trail Making Test as an indicator of organic brain damage. Perceptual and Motor Skills, 1(8), 271-276. doi: 10.2466/PMS.8.7.271-276

Reitan, R. M., \& Wolfson, D. (1985). The HalsteadReitan Neuropsycholgical Test Battery: Therapy and clinical interpretation. Tucson, AZ: Neuropsychological Press.

Sabbath, E. L., Gutierrez, L.-A., Okechukwu, C. A., Singh-Manoux, A., Amieva, H., Goldberg, M., ...Berr, C. (2014). Time may not fully attenuate solvent-associated cognitive deficits in highly exposed workers. Neurology, 82(19), 17161723. doi: 10.1212/WNL.0000000000000413

Saygun, M., Ekici, A., Muluk, N. B., Çakmak, A., Pinar, T., Dag, E., \& Ekici, M. (2012). Effects of long-term low-level solvent exposure on cognitive function. Clinical \& Investigative Medicine, 35(4), E190-E205. Retrieved from http://cimonline.ca/index.php/cim/article/viewFile/17148/14096

Soldin, O. P., \& Mattison, D. R. (2009). Sex differences in pharmacokinetics and pharmacodynamics. Clinical Pharmacokinetics, 48(3), 143-157. doi: 10.2165/00003088-200948030-00001

Song, H., Yu, I.T.-S., \& Lao, X.Q. (2015). Neurobehavioral effects of occupational exposure to organic solvents among male printing workers in Hong Kong. Archives Environmental Occupational Health, 70, 147-153. doi: 10.1080/19338244.2013.828676

Spyker, J. M. (1979). Assessing the impact of low level chemicals on development: Behavioral and latent effects. Federation proceedings, 34(9), 1835-1844. Retrieved from https://www.ncbi. nlm.nih.gov/pubmed/1097268

Tang, C. Y., Carpenter, D. M., Eaves, E. L., Ng, J., Ganeshalingam, N., Weisel, C., ...Fiedler, N. L. (2011). Occupational solvent exposure and brain function: An fMRI Study. Environmental Health Perspectives, 119(7), 908-913. doi: 10.1289/ehp.1002529

Thetkathuek, A., Jaidee, W., Saowakhontha, S., \& Ekburanawat, W. (2015). Neuropsychological symptoms among workers exposed to toluene and xylene in two paint manufacturing factories in Eastern Thailand. Advances in Preventive Medicine. doi:10.1155/2015/183728

Tomicic, C., \& Vernez, D. (2014). Sex differences in urinary levels of several biological indicators of exposure: A simulation study using a compartmental-based toxicokinetic model. Journal Occupational Environmental Hygiene, 11(6), 377-387. doi: 10.1080/15459624.2013.875180

Tseng, L. A., Delmonico, M. J., Visser, M., Boudreau, R. M., Goodpaster, B. H., Schwartz, A. V., ...Newman, A. B. (2014). Body composition explains sex differential in physical performance among older adults. The Journals of Gerontology: Series A, 69(1), 93-100. doi: 10.1093/gerona/glt027

Vahter, M., Gochfeld, M., Casati, B., Thiruchelvam, M., Falkfilippson, A., Kavlock, R., ... Coryslechta, D. (2007). Implications of gender differences for human health risk assessment and toxicology. Environmental Research, 104(1), 70-84. doi: 10.1016/j.envres.2006.10.001

Visser, I., Lavini, C., Booij, J., Reneman, L., Majoie, C., Boer, A. G.,... Den Heeten, G. J. (2008). Cerebral impairment in chronic solvent-induced encephalopathy. Annals Neurology, 63(5), 572580. doi: 10.1002/ana.21364

Weiss, B. (2012). The intersection of neurotoxicology and endocrine disruption. NeuroToxicology, 33(6), 1410-1419. doi: 10.1016/j.neuro.2012.05.014

Wood, R. L., \& Liossi, C. (2005). Long-term neuropsychological impact of brief occupational exposure to organic solvents. Archives of Clinical Neuropsychology, 20(5), 655-665. doi: 10.1016/j.acn.2005.01.003 
Zavalić, M., Mandić, Z., Turk, R., Bogadi-Sare, A., \& Plavec, D. (1998). Quantitative assessment of color vision impairment in workers exposed to toluene. American Journal of Industrial Medicine, 3c3(3), 297-304. Retrieved from https:// www.ncbi.nlm.nih.gov/pubmed/9481429

Zhong, Z., Zeng, T., Xie, K., Zhang, C., Chen, J., Bi, Y., \& Zhao, X. (2013). Elevation of 4-hydroxynonenal and malondialdehyde modified protein levels in cerebral cortex with cognitive dysfunction in rats exposed to 1-bromopropane. Toxicology, 306, 16-23. doi: 10.1016/j. tox.2013.01.022

Recebido: $28 / 04 / 2017$

$1^{a}$ revisão: $18 / 02 / 2018$

Aceite final: $26 / 02 / 2018$ 